\section{HCV in a Dialysis Center}

Schneeberger and colleagues have described the transmission of hepatitis $\mathrm{C}$ virus (HCV) in a dialysis center in The Netherlands.

A cluster of four almost identical HCV isolates (genotype 2a) was found in the dialysis center. Within a period of 1 year, another cluster of four HCV-infected dialysis patients was detected in the same center. These four isolates were almost identical to a fifth isolate, genotype $2 \mathrm{~b}$, found in the earlier study from another patient dialyzed in the same unit. It was observed that possibly contaminating procedures were not strictly separated. Some of the shared medical equipment was not disinfected, but only cleaned. Also, bloodcontaminated gloves might have played a role in the transmission of HCV. It was concluded that nosocomial transmission played an important role in the epidemiology of $\mathrm{HCV}$ in these dialysis patients. Shared medical equipment and blood-contaminated gloves may constitute a potential route of transmission. There is a need for stringent implementation and regular auditing of infection control measures.

FROM: Schneeberger PM, Doorenbos CJ, van der Vliet W, Bergervoet P, Quint W, van Doorn LJ. Nosocomial transmission of hepatitis $\mathrm{C}$ virus in a Dutch dialysis center. Ned Tijdschr Geneeskd 1999;143:801-806.

\section{Emergence of Antibiotic-Resistant Pseudomonas aeruginosa}

Carmeli and colleagues from Beth Israel Deaconess Medical Center and Harvard Medical School compared the risks of emergence of resistance associated with four antipseudomonal agents: ciprofloxacin, ceftazidime, imipenem, and piperacillin. A total of 271 patients (followed for 3,810 days) with infections due to Pseudomonas aeruginosa were treated with the study agents. Resistance emerged in 28 patients (10.2\%). Adjusted hazard ratios for the emergence of resistance were: ceftazidime, 0.7 ; ciprofloxacin, 0.8; imipenem, 2.8; and piperacillin, 1.7. Hazard ratios for emergence of resistance to each individual agent associated with treatment with the same agent were: ceftazidime, 0.8 ; ciprofloxacin, 9.2; imipenem, 4.4; and piperacillin, 5.2. It was concluded that there were differences among antibiotics in the likelihood that their use would allow emergence of resistance in $P$ aeruginosa. Ceftazidime was associated with the lowest risk, and imipenem had the highest risk.

FROM: Carmeli Y, Troillet N, Eliopoulos GM, Samore MH. Emergence of antibiotic-resistant Pseudomonas aeruginosa: comparison of risks associated with different antipseudomonal agents. Antimicrob Agents Chemother 1999;43:1379-1382.

\section{MRSA Carriers in a Nursery}

Mitsuda and coworkers from the Department of Pediatrics, School of Medicine, Yokohama City
University, Japan, examined two persistent methicillinresistant Staphylococcus aureus (MRSA)-carrier nurses in a maternity hospital to elucidate the transmission of MRSA from healthcare providers to newborn infants and to the nurses' own families. Genotyping of the MRSA strains was performed by analyzing genomic DNA restriction fragment-length polymorphisms from pulsedfield gel electrophoresis. The children of these nurses were carrying MRSA strains genotypically identical to those of their mothers. Both MRSA carrier families remained asymptomatic over a 2-year follow-up period. Eradication of nasal MRSA carriage from the two nurses resulted in declining MRSA carriage rates among infants in the nursery. The authors hypothesized that healthcare providers may become transient or persistent MRSA carriers while working in hospitals where MRSA is endemic. They may then become a source of infection for patients, as well as their own families. They recommend that healthcare providers should be examined for MRSA if an MRSA epidemic occurs in a hospital. The families of any such carriers should also be examined for MRSA.

FROM: Mitsuda T, Arai K, Ibe M, Imagawa T, Tomono $\mathrm{N}$, Yokota $\mathrm{S}$. The influence of methicillin-resistant Staphylococcus aureus (MRSA) carriers in a nursery and transmission of MRSA to their households. $J$ Hosp Infect 1999;42:45-51.

\section{MRSA in Nursing Home Residents}

Niclaes and colleagues from the University of Leuven, Belgium, conducted a prospective cohort study with 1-year follow-up to evaluate the relation between methicillin-resistant Staphylococcus aureus (MRSA) carriage and mortality, likelihood of hospitalization, and functional status in residents of a nursing home for the elderly. Included were all 447 residents living in the home in early June 1994. Swabs were taken from nose, throat, and perineum, and additional swabs (sputum, urine, or wounds) were taken when indicated. The relative risk (RR) of dying within 6 months in MRSA carriers compared to noncarriers was 2.29 (95\% confidence interval $\left.\left[\mathrm{CI}_{95}\right], 1.04-5.04\right)$. This $\mathrm{RR}$ remained stable (1.57-2.40) after adjustment for covariables using Mantel-Haenszel stratified analysis. After 1 year, the RR was reduced to $1.30\left(\mathrm{CI}_{95}, 0.65-2.58\right)$. Univariate survival analysis confirmed a difference in survival between carriers and noncarriers after 6 months (log-rank $P=.04$ ) and no difference after 1 year. Cox regression analysis resulted in a hazard ratio for dying within 6 months of $1.73\left(\mathrm{CI}_{95}, 0.72\right.$ 4.17). No relation was found between MRSA carriage and either likelihood of hospitalization or indicators of functional status. These results are compatible with a possible relation between 6-months' mortality and MRSA carriage in nursing home patients.

FROM: Niclaes L, Buntinx F, Banuro F, Lesaffre E, Heyrman J. Consequences of MRSA carriage in nursing home residents. J Epidemiol Infect 1999;122:235-239. 